Elsevier Editorial System(tm) for Current Opinion in Structural Biology Manuscript Draft

Manuscript Number:

Title: Arginine and Nitrogen Storage

Article Type: Catalysis and regulation

Corresponding Author: Dr Ignasi Fita,

Corresponding Author's Institution:

First Author: José L Llácer

Order of Authors: José L Llácer; Ignasi Fita; Vicente Rubio 


\title{
Arginine and Nitrogen Storage
}

\author{
José L. Llacer ${ }^{1}$, Ignacio Fita ${ }^{2}$ and Vicente Rubio ${ }^{1}$
}

\section{Addresses}

${ }^{1}$ Instituto de Biomedicina de Valencia-Consejo Superior de Investigaciones Cientificas (IBV-CSIC) and Centro de Investigación Biomédica en Red de Enfermedades Raras (CIBERER-ISCIII), Jaime Roig 11, Valencia 46010, Spain.

${ }^{2}$ Instituto de Biología Molecular de Barcelona-Consejo Superior de Investigaciones Científicas (IBMB-CSIC) and Institute for Research in Biomedicine, Josep Samitier 15, Parc Cientific, Barcelona 08028, Spain.

\section{Corresponding authors:}

Fita, Ignacio (ifrcri@ibmb.csic.es)

Rubio, Vicente (rubio@ibv.csic.es)

\section{Summary}

When nitrogen is abundant, prokaryotic and eukaryotic oxygen-producing photosynthetic organisms store nitrogen as arginine, by relieving feedback inhibition of the arginine biosynthesis controlling enzyme, N-acetylglutamate kinase (NAGK). The signalling protein PII, an ancient and widely distributed nitrogen/carbon/ADP/ATP sensor, mediates feed-back inhibition relief of NAGK by binding to this enzyme. PII phosphorylation or PII binding of ADP or 2-oxoglutarate prevent PII-NAGK complex formation. Crystal structures of NAGK and of cyanobacterial and plant PII and of the corresponding PII-NAGK complexes have been recently determined. In these complexes, two polar PII trimers sandwich one ring-like NAGK hexamer. Each PII subunit contacts one NAGK subunit, triggering a symmetry-restricted narrowing of the 
NAGK ring, with concomitant adoption by the arginine sites of a low-affinity conformation.

\section{Introduction}

Nitrogen assimilation is a requisite for growth. The nitrogen can be supplied as ammonia, nitrate, dinitrogen or amino acids $\left[1,2^{\circ}\right]$. Ammonia incorporation is cheap in terms of energy and reducing power, being generally effected (Figure 1a) by the combined action of glutamine synthetase (GS) and glutamate synthase (GOGAT), using 2-oxoglutarate (2OG) to generate glutamate [1-3], that then provides the nitrogen for making other amino acids [4]. Pioneering studies of GS regulation [5] revealed a key controlling protein that was called PII because it was peak II in a gel filtration column [6]. PII, a very ancient signalling protein that is widely distributed throughout all domains of life but that is absent from animals $\left[1,2^{\circ}, 7,8^{* *}\right]$, senses and integrates nitrogen and carbon abundance and energy status information, by binding $2 \mathrm{OG}$ and ATP/ADP and, in many organisms, by being either phosphorylated, uridylylated or adenylylated [9-11].

PII interacts with and influences many protein targets, including enzymes, channels and regulatory proteins $\left[1,2^{\bullet}, 7,8^{\bullet}, 12,13\right]$. Crystal structures of PII from various sources were obtained [14-19*3 but little was known about how PII exerts its effects. Very recently, crystal structures of complexes of PII with the ammonium channel AmtB (Figure 1 b) $\left[20^{* *}, 21^{* *}\right]$ or with the enzyme NAGK $\left[22^{*}, 23^{*}\right]$ have been determined. In the complex with AmtB, the large and flexible T-loop of PII occludes the cytoplasmic opening of AmtB (Figure 1c) by means of its tip residue Arg47 (Figure 1d), blocking channel operation. The present review will concentrate on the other complex, with the enzyme of arginine biosynthesis N-acetyl-L-glutamate kinase (NAGK). The structure of 
this complex reveals a highly sophisticated activation mechanism that renders possible massive nitrogen storage as arginine. For recent reviews on other aspects of PII function, see $\left[1,2^{\bullet}, 7,8^{\bullet \bullet}, 24-26\right]$

\section{Control of nitrogen storage as arginine by the signalling protein PII}

NAGK was recently recognized in cyanobacteria and plants as a PII target [2732]. These organisms store nitrogen as arginine (Figure 1a), which is incorporated into proteins such as seed proteins $[33,34]$ or in arginine-rich copolymers [35]. Arginine is an ideal nitrogen store because it is nitrogen-rich, its incorporation into proteins minimizes its osmotic impact, and its nitrogen is easily mobilized [36]. Since arginine synthesis is feed-back inhibited at the NAGK level [34,37,38], this enzyme should be released from inhibition for build-up of large arginine stores. In oxygenic phototrophs, PII triggers this effect on NAGK by binding to the enzyme when nitrogen is abundant [27-32]. When nitrogen is scarce, $20 \mathrm{O}$ accumulates because of reduced flow through GS/GOGAT and binds to PII in the presence of ATP, promoting PII-NAGK complex dissociation [29]. ADP binding to PII, and 2OG-promoted PII phosphorylation at Ser49 also prevent PII-NAGK complex formation in cyanobacteria [27-29].

\section{Arginine-sensitive NAGKs are allosteric switches operated by arginine}

The first NAGK crystal structure, of the arginine-insensitive Escherichia coli enzyme $[39,40]$ revealed a homodimer having as backbone a 16 -stranded $\beta$-sheet spanning both subunits (Figure 2a). Each 258-residue subunit presents the amino acid kinase fold (PFam PF00696; http://pfam.sanger.ac.uk): an open $\alpha_{3} \beta_{8} \alpha_{4}$ sandwich composed of $\mathrm{N}$ - and $\mathrm{C}$-domains that host, respectively, on the C-edge of the central, 
mainly parallel, $\beta$-sheet, the sites for the substrates N-acetyl-L-glutamate (NAG) and ATP.

Arginine-sensitive NAGKs were known to be hexameric [29,41,42]. The recent determination of the first crystal structures of these enzymes, free [43] or, more recently, PII-complexed $\left[22^{* *}, 23^{* *}\right]$, has shown them to be ring-like hexameric trimers

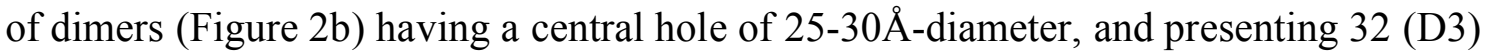
molecular symmetry $[22 *, 23 *, 43]$. The ring is formed by linking three E. coli NAGKlike dimers through the interlacing of a $\mathrm{N}$-terminal $\alpha$-helix (the $\mathrm{N}$-helix) that is not found in arginine-insensitive NAGKs. Arginine binds flat and extended near the interdimeric boundary (Figures $2 \mathrm{~b}$ and $2 \mathrm{e}$ ) in a cavity formed in the C-domain of each subunit between the $\alpha_{3}$ layer, the $\beta$ sheet, and the $\mathrm{C}$-terminal part of the $\mathrm{N}$-helix after the kink (see below figures $4 \mathrm{f}$ and $4 \mathrm{~g}$ ). In agreement with the remoteness of this site relative to the substrate sites, arginine inhibits the reaction indirectly (Figure 2c), by increasing the separation between the C-and N-domains hosting the ATP and NAG sites [43]. The changes triggered by arginine inhibition were associated with changes in the tilt of the enzyme dimers within the hexamer (Figures 2d-e), rendering the hexameric ring wider and thinner (Figures 2c, 2d and 2e) [43].

The interlaced $\mathrm{N}$-helices connect in the hexamer the arginine sites from adjacent dimers [43], and thus these helices and the hexameric organization are essential features for making NAGK an arginine-operated switch exhibiting sharp sigmoid arginine inhibition kinetics $\left[29,41,44^{\circ}\right]$. Thus, at arginine concentrations below a certain threshold, the enzyme is not inhibited, allowing arginine synthesis. However, because of the sigmoid character of the inhibition, the enzyme is inhibited quite abruptly when this threshold arginine concentration is exceeded, stopping arginine synthesis (Figure $2 \mathrm{f}$ ). 


\section{PII structure}

PII proteins are homotrimers of $12-13-\mathrm{kDa}$ subunits having a $\beta \alpha \beta \beta \alpha \beta$ subunit topology, with $\alpha$ helices looking outward and the $\beta$ sheet inward and providing the intersubunit interactions (Figures $3 \mathrm{a}$ and $3 \mathrm{~b}$ ) [14-19“*. The trimer body is hemispheric, from which two short loops (the B- and C-loops) and a large and highly flexible loop called the T-loop emerge from each subunit. The T-loop residues S49 and Y51 are, respectively, the phosphorylation and uridylylation sites in cyanobacteria and proteobacteria. When modified, the PII proteins cannot interact with their respective NAGK or AmtB targets [8*3. ADP and MgATP bind to PII in a crevice between adjacent subunits in the convex surface of the PII body, at the base of the T-loop (Figures $3 \mathrm{a}$ and $3 \mathrm{~b}$ ) $\left[15,19{ }^{*}-21^{*}, 23^{*}\right]$. MgATP binding is associated with a compact conformation of the $\mathrm{T}$-loop resembling a bent finger in which the $\mathrm{Mg}$ interactions play a crucial role (Figures 3b-3d), whereas ADP, bound without visible Mg ions at the same site, favours an extended T-loop conformation [19*3 (Figures 3a and 3c). Only in Methanococcus jannaschii PII one 2OG molecule has been found binding to one T-loop that also had MgATP bound [19" (Figure 3d), in agreement with the well known requirement of ATP for $2 \mathrm{OG}$ binding [8*0. This T-loop was in the compact conformation $\left[19^{\circ}\right]$, and thus the site for $2 \mathrm{OG}$ may be formed only when the T-loop adopts its compact conformation.

\section{Effects of PII binding to NAGK}

The effects of PII on NAGK have been characterized functionally best in the cyanobacteria Synechococcus elongatus strain PCC7942 and in the plant Arabidopsis thaliana $[29,32]$, the same species in which the crystal structures of the complexes have been determined $\left[22^{*}, 23^{*}\right]$. The NAGKs from both species present some functional 
differences, and the effects of PII appear not to be identical, perhaps reflecting differences in the adaptive requirements in the cytoplasm of a free-living cyanobacterium and in plant chloroplasts, where PII and NAGK are found [30,45]. S.elongatus NAGK is virtually inactive in the absence of PII, having low $\mathrm{V}_{\max }$, high $\mathrm{K}_{\mathrm{m}}$ for NAG $(\sim 30 \mathrm{mM})$ [29] and requiring low arginine concentrations $\left(\mathrm{I}_{0.5} \sim 30 \mu \mathrm{M}\right)$ for inhibition [22*] (Figure 2f). A.thaliana NAGK is highly active, having, relative to S.elongatus $\mathrm{NAGK}, \sim 3$-fold higher $\mathrm{V}_{\max }$ and $\sim 4$-fold lower $\mathrm{K}_{\mathrm{m}}$ for $\mathrm{NAG}$, and requiring one order of magnitude higher arginine concentrations for inhibition [32]. In S.elongatus, PII activates NAGK up to $\sim 40$-fold by increasing 2-4-fold $\mathrm{V}_{\max }$, by decreasing $\sim 10$-fold the $\mathrm{K}_{\mathrm{m}}$ for $\mathrm{NAG}$, and by augmenting $\sim 15$-fold the arginine concentration required for inhibition [29] (Figure 2f). In contrast, in A.thaliana, PII activates NAGK up to 5-fold only, not affecting the $\mathrm{K}_{\mathrm{m}}$ for NAG, increasing marginally $(\sim 30 \%) \mathrm{V}_{\max }$ and triggering a mere $\sim 3$-fold increase in the concentrations of arginine needed for inhibition [32]. ADP and, under appropriate conditions, 2OG, dissociated the S.elongatus PII-NAGK complex [29], but these ligands were not found to dissociate in vitro the isolated complex from A.thaliana [32].

\section{Known structures of PII-NAGK complexes}

The reported crystal structures of the S.elongatus and A.thaliana complexes are highly similar $\left[22^{*}, 23^{*}\right]$ although only NAG was bound to the S.elongatus complex, whereas, in A.thaliana, NAG, ADP and arginine were bound to NAGK and MgATP was bound to PII. We will describe first the S.elongatus complex, and then we will highlight relevant differential traits of the A.thaliana complex. These complexes are the genuine ones formed in vivo: their stoichiometry is the same demonstrated for the $S$. elongatus complex in solution, the interacting residues include those conserved 
exclusively in oxygenic phototrophs (the organisms in which this complex is formed), and structure-directed mutagenesis of PII and NAGK triggered the expected effects in vivo (yeast two-hybrid assays) and in functional assays of complex formation [22“].

\section{The structure of the S.elongatus PII-NAGK complex [22**]}

The complex (Figure 4a) consists of one NAGK hexamer sandwiched between two PII trimers oriented with their convex surface pointing outwards. PII is not packed tightly on NAGK, interacting with NAGK mainly through its T- and B-loops (Figures $4 \mathrm{~b}$ and $4 \mathrm{c}$ ). Each PII subunit interacts with one NAGK subunit only, contacting the $\alpha_{4}$ layer near its connection with the central $\beta$ sheet $\mathrm{N}$-edge, far from both the arginine site and the sites for the substrates (Figure 4d). The T-loop is in the compact form resembling a bent finger (Figure 3b), explaining why ADP, by favouring an extended Tloop conformation, disassembles the complex [29]. The T-loop tip is inserted in the ridge between the NAGK N- and C-domains, interacting through several polar bonds with the N-domain and with Gln258 from the C-domain (Figures $4 \mathrm{~d}$ and 4e). An ion pair network centred in PII reaches close to the NAG site of NAGK (Figure 4e), favouring a high affinity form of this site, explaining the decreased $\mathrm{K}_{\mathrm{m}}$ for NAG. The hydroxyl of Ser49 is centrally involved in the interactions with NAGK, explaining the lack of complex formation upon Ser49 phosphorylation. If $20 \mathrm{OG}$ binds to PII in S.elongatus as in M.jannaschii [19“], 2OG-triggered complex disassembly must be due to the negative charge contributed by $2 \mathrm{OG}$, rather than to inappropriate T-loop structure or to steric clashes.

The numerous T-loop contacts with the N-domain fix this domain and the NAGK dimers in a position [22“] that favours a high dimer tilt and, consequently, an arginine-free-like orientation of the N-helix [43]. This conformation differs from the 
arginine-inhibited NAGK form, characterized by low-tilt dimers, a widened ring, and a characteristic N-helix orientation (Figure 2c) [43]. Thus, PII binding favours the active, low affinity form (for arginine) of the NAGK hexamer. These global NAGK ring changes fit the principle of preservation of symmetry in domain movements [46]. This principle predicts for NAGK that, in the twofold axis-related subunits of different dimers, the cycle of opening and closing of the active centres that is consubstantial with enzyme catalysis should be facilitated by coordinating the movements of the two subunits to preserve the twofold symmetry. This necessarily requires that the tilt and the distance of the dimers from the threefold axis is changed, as observed, because of the restrictions imposed by the threefold symmetry of the D3 point group.

Interactions not mediated by the T-loop, involving the B-loop and the $\beta 1-\alpha 1$ junction (particularly Phe11) close to the flat face of the PII body, target the C-domain of NAGK, possibly accounting for the reported [22*3 C-domain displacement of $\sim 2 \AA$ towards PII that appears to contribute, together with the inappropriate (for arginine binding) N-helix orientation, to the observed arginine site enlargement and the low affinity for arginine (compare figures Figures $4 \mathrm{f}$ and $4 \mathrm{~h}$ ).

\section{The A.thaliana PII-NAGK complex [23“]}

Although MgATP was not added and $10 \mathrm{mM}$ ADP was present in the crystallization mixture [23*], the complex contains MgATP bound to PII similarly as in M.jannaschii PII [19"], reflecting the previously demonstrated [47] high affinity of A.thaliana PII for MgATP (Figure 3b). Thus, the reported lack of complex dissociation by ADP [32] may have reflected the lack of displacement of PII-bound MgATP. The lack of effect of PII binding on the $\mathrm{K}_{\mathrm{m}}$ for NAG of A.thaliana NAGK [32] is explained by the exclusion of the NAG site from the ion-pair network formed upon T-loop 
binding, because of the substitution by Asn of the corresponding Asp found in S.elongatus NAGK [22“]. Trp22 (corresponding to Phe11 of S.elongatus PII) was the only non-T-loop residue reported [23"*] to interact with NAGK, but, actually, Lys 240 interacts in one subunit of A.thaliana NAGK (see PDB file 2rd5) with Glu97 of PII, similarly to Arg233 of NAGK with Glu85 in the complex of S.elongatus. In the A.thaliana complex all the NAGK active centres contain bound NAG and ADP (Figure 4d), and they exhibit in three subunits a closed conformation that possibly may be equated with a catalytically active form. The movement causing this closing of the active centre is a $11^{\circ}$-rotation around a hinge located at the interdomain boundary in the NAGK subunit, near the site at which the T-loop contacts NAGK [23*]. Thus, the stimulatory effect of PII on the kinetics of arginine-inhibited NAGK has been attributed [23“] to PII-limitation of the degree of opening and closing of the active site cleft, in opposition to the domain-separating inhibitory effect exerted by arginine. Arginine was present at high concentration $(50 \mathrm{mM})$ in the crystallization mixture and was also found binding in all the subunits, but, as expected from the overall changes triggered by the binding of PII, the N-helix is inappropriately oriented for high affinity binding of arginine, yielding a low-affinity conformation of the arginine site (Figure 4g).

\section{Conclusions and prospects}

Despite the high similarities of the corresponding complexes, there are substantial differences in the sensitivity to PII of cyanobacterial and A.thaliana NAGKs. Perhaps in other photosynthetic eukaryotes expected to experience more changes in nitrogen supply than higher plants, such as unicellular algae or even multicellular algae and lower plants, the effects of PII on NAGK might be closer to those in cyanobacteria. Therefore, the PII-NAGK system should be investigated in lower photosynthetic 
eukaryotes. More work is also needed on A.thaliana to clarify whether $2 \mathrm{OG}$ regulates the PII-NAGK complex, since $2 \mathrm{OG}$ is a low-nitrogen abundance signal in plants [48] and was found to bind to A.thaliana PII [47]. It is therefore surprising the lack of effect of $2 \mathrm{OG}$ on the PII-NAGK complex, as reported [32]. Another question deserving further work is the possibility that nitrogen accumulation as arginine could take place in other taxonomic groups, given the very widespread co-occurrence in the same organism of PII [1] and hexameric arginine-sensitive NAGK [34,37,38,41]. Although this possibility is not favoured by the exclusive conservation in photosynthetic organisms of a subset of the residues found to interact in PII-NAGK complexes $\left[22^{* *}, 23^{* *}\right]$, the possibility of the existence of different complementary sets of interacting residues in other taxonomic groups cannot be excluded. It is also important to determine the structure of novel PII complexes with other targets [49], to clarify whether the T-loop conformations observed in the PII-AmtB and PII-NAGK complexes are the only two standard conformations used in the interactions of PII or whether the T-loop conformation is tailored specifically to each target, resulting in a very diverse array of modes of regulating the functionality of the different targets.

\section{Acknowledgements}

This work was supported by Grants BFU2004-05159 and BFU2005-08686-C02-01. J.L.L. is a FPI fellow of the Spanish Ministry of Education and Science. We thank A. Contreras and K. Forchhammer for critical reading of the manuscript, and S. RamónMaiques with help with the figures. 


\section{References and recommended reading}

Papers of particular interest, published within the annual period of review, have been highlighted as:

- of special interest

•• of outstanding interest

1. Arcondeguy $\mathrm{T}$, Jack R, Merrick M: PII signal transduction proteins, pivotal players in microbial nitrogen control. Microbiol Mol Biol Rev 2001, 65:80-105.

2. Leigh JA, Dodsworth JA: Nitrogen regulation in bacteria and archaea. Annu Rev - $\quad$ Microbiol 2007, 61:349-377.

This review of nitrogen regulation in prokaryotes gives a good updated picture of the elements known to be involved in this regulation. The numerous illustrations provided of reactions and regulation mechanisms give clear schematics that are very helpful for understanding the regulation.

3. Suzuki A, Knaff DB: Glutamate synthase: structural, mechanistic and regulatory properties, and role in the amino acid metabolism. Photosynth Res 2005, 83:191-217.

4. Bender DA: Amino Acid Metabolism. John Wiley \& Sons; 1985.

5. Stadtman ER: The story of glutamine synthetase regulation. J Biol Chem 2001, 276:44357-44364. 
6. Shapiro BM: The glutamine synthetase deadenylylating enzyme system from Escherichia coli. Resolution into two components, specific nucleotide stimulation, and cofactor requirements. Biochemistry 1969, 8:659-670.

7. Ninfa AJ, Jiang P: PII signal transduction proteins: sensors of alphaketoglutarate that regulate nitrogen metabolism. Curr Opin Microbiol 2005, 8:168-173.

8. Forchhammer $\mathrm{K}$ : $\mathbf{P}(\mathrm{II})$ signal transducers: novel functional and structural •• $\quad$ insights. Trends Microbiol 2008, 16:65-72.

Very clear and updated review of our present-day knowledge of PII protein functions. It integrates the existing structural knowledge at the moment of writing. It has very clear schemes to illustrate the present understanding of three different modes of negative regulation exerted by PII. It also schematizes in a very clear way the PII-NAGK system.

9. Adler SP, Purich D, Stadtman ER: Cascade control of Escherichia coli glutamine synthetase. Properties of the PII regulatory protein and the uridylyltransferase-uridylyl-removing enzyme. J Biol Chem 1975, 250:62646272.

10. Forchhammer K, Tandeau de Marsac N: The PII protein in the cyanobacterium Synechococcus sp. strain PCC 7942 is modified by serine phosphorylation and signals the cellular N-status. J Bacteriol 1994, 176:84-91. 
11. Hesketh A, Fink D, Gust B, Rexer HU, Scheel B, Chater K, Wohlleben W, Engels A: The GInD and GlnK homologues of Streptomyces coelicolor A3(2) are functionally dissimilar to their nitrogen regulatory system counterparts from enteric bacteria. Mol Microbiol 2002, 46:319-330.

12. Espinosa J, Forchhammer K, Burillo S, Contreras A: Interaction network in cyanobacterial nitrogen regulation: PipX, a protein that interacts in a 2oxoglutarate dependent manner with PII and NtcA. Mol Microbiol 2006, 61:457-469.

13. Osanai T, Sato S, Tabata S, Tanaka K: Identification of PamA as a PII-binding membrane protein important in nitrogen-related and sugar-catabolic gene expression in Synechocystis sp. PCC 6803. J Biol Chem 2005, 280:3468434690

14. Cheah E, Carr PD, Suffolk PM, Vasudevan SG, Dixon NE, Ollis DL: Structure of the Escherichia coli signal transducing protein PII. Structure 1994, 2:981990.

15. Xu Y, Cheah E, Carr PD, van Heeswijk WC, Westerhoff HV, Vasudevan SG, Ollis DL: GInK, a PII-homologue: structure reveals ATP binding site and indicates how the T-loops may be involved in molecular recognition. $\mathrm{J} \mathrm{Mol}$ Biol 1998, 282:149-165 
16. Xu Y, Carr PD, Clancy P, Garcia-Dominguez M, Forchhammer K, Florencio F, Vasudevan SG, Tandeau De Marsac N, Ollis DL: The structures of the PII proteins from the cyanobacteria Synechococcus sp. PCC 7942 and Synechocystis sp. PCC 6803. Acta Crystallogr D 2003, 59:2183-2190.

17. Sakai H, Wang H, Takemoto-Hori C, Kaminishi T, Yamaguchi H, Kamewari Y, Terada T, Kuramitsu S, Shirouzu M, Yokoyama S: Crystal structures of the signal transducing protein GInK from Thermus thermophilus HB8. J Struct Biol 2005, 149:99-110.

18. Mizuno Y, Berenger B, Moorhead GB, Ng KK Crystal structure of Arabidopsis

- $\quad$ PII reveals novel structural elements unique to plants. Biochemistry 2007, 46:1477-1483.

This is the first crystal structure of a plant PII protein. The structure, at $1.9 \AA$ resolution, presents the characteristic PII fold and trimeric architecture and has the T-loop disordered, but it reveals some plant-specific specializations, including extensions of unknown signification at both ends of the PII polypeptide.

19. Yildiz O, Kalthoff C, Raunser S, Kuhlbrandt W: Structure of GInK1 with bound

-• $\quad$ effectors indicates regulatory mechanism for ammonia uptake. $E M B O J 2007$, 26:589-599.

This study shows that the PII protein of $M$. jannaschii GlnK1 forms a complex with the channel AmtB, revealing the influence of effectors, particularly 2OG, on complex formation. It also visualizes $\mathrm{AmtB}$ and the GlnK1-AmtB complex by electron microscopy. Finally, it reports crystal structures of GlnK1 with MgATP or with ADP, 
and it provides the first snapshot of the effector $2 \mathrm{OG}$ binding to a PII protein. These studies demonstrate that MgATP and ADP are associated, respectively, with an extended conformation and a compact novel conformation of the T-loop. The finding of $2 \mathrm{OG}$ bound to the compact conformation of the T-loop having bound MgATP indicates that the site for $2 \mathrm{OG}$ is formed when MgATP is bound, explaining the cooperative binding of ATP and $2 \mathrm{OG}$ to PII. The dissociation of the GlnK1-AmtB complex triggered by $20 \mathrm{OG}$ is explained on the basis of the increase in the exposed negative charge of the T-loop.

20. Gruswitz F, O'Connell J III, Stroud RM: Inhibitory complex of the -• transmembrane ammonia channel, AmtB, and the cytosolic regulatory protein, GlnK, at 1.96 A. Proc Natl Acad Sci USA 2007, 104:42-47.

This study and $\left[21^{\circ}\right]$ are the first to report the structure of a PII-target protein complex at atomic resolution. Both studies reveal that GlnK (a PII protein of E. coli) interacts with AmtB almost exclusively via the T-loop, blocking the cytoplasmic opening of the channel by inserting into it the T-loop tip residue Arg47. ADP was found binding to PII in both studies, supporting the role of this nucleotide in promoting the extended T-loop conformation $\left[1^{\circ}\right]$. The structures explain also why the uridylylation of the T-loop residue Tyr51 prevents PII-AmtB complex formation.

21. Conroy MJ, Durand A, Lupo D, Li XD, Bullough PA, Winkler FK, Merrick M: The

-• crystal structure of the Escherichia coli AmtB-GInK complex reveals how GInK regulates the ammonia channel. Proc Natl Acad Sci USA 2007, 104:1213-1218.

See annotation for $\left[20^{\circ}\right]$ 
22. Llácer JL, Contreras A, Forchhammer K, Marco-Marín C, Gil-Ortiz F, Maldonado

- $\quad$ R, Fita I, Rubio V: The crystal structure of the complex of PII and acetylglutamate kinase reveals how PII controls the storage of nitrogen as arginine. Proc Natl Acad Sci USA 2007, 104:17644-17649.

This study and $\left[23^{\circ}\right]$ report the first crystal structures of PII-NAGK complexes. The cyanobacterial proteins from S. elongatus PCC 7942 are used in this study reporting the structure of the complex at $2.75-\AA$ resolution and containing NAG bound to NAGK. PII presents the compact conformation (see [19*0) although no MgATP is bound. It is concluded that the compact T-loop conformation is selected by NAGK. The central participation in the interactions between PII and NAG of the T-loop residue Ser49 explains complex dissociation by Ser49 phosphorylation. The complex-dissociating effect of $20 \mathrm{OG}$ is attributed to negative charge repulsion, and it is predicted to be dependent on the ionic composition of the medium. The decreased $\mathrm{K}_{\mathrm{m}}$ of NAGK for NAG within the complex is attributed to PII-triggered NAG site conformational changes. NAGK relief from arginine inhibition is due to changes in the arginine site resulting from symmetry-determined changes in the overall shape of the NAGK hexamer as well as to local effects.

23. Mizuno Y, Moorhead GB, Ng KK: Structural basis for the regulation of $\mathbf{N}$ -

-• acetylglutamate kinase by PII in Arabidopsis thaliana. J Biol Chem 2007, 282:35733-35740.

See also annotation for [22** . This study reports the crystal structure at $2.5 \AA$-resolution of the plant PII-NAGK complex. This complex, and the cyanobacterial complex reported in $\left[22^{\circ}\right]$ are highly similar. The crystals contain MgATP bound to PII and 
NAG, ADP and arginine bound to NAGK. The site for the arginine inhibitor is in a lowaffinity form because of the orientation of the N-terminal helices that interconnect the three dimers in the hexamer. Both NAGK subunits in the asymmetric unit exhibit different degrees of active centre opening. The movement causing this active centre change is a rotation around a hinge located at the boundary between both domains of each NAGK subunit, near the site at which the T-loop contacts NAGK. Thus, the stimulatory effect of PII on the kinetics of arginine-inhibited NAGK is attributed to a PII-limited degree of opening and closing of the active site cleft, in opposition to a domain-separating inhibitory effect exerted by arginine.

24. Dixon R, Kahn D: Genetic regulation of biological nitrogen fixation. Nat Rev Microbiol 2004, 2:621-631.

25. Commichau FM, Forchhammer K, Stülke J: Regulatory links between carbon and nitrogen metabolism. Curr Opin Microbiol 2006, 9:167-172.

26. Muro-Pastor MI, Reyes JC, Florencio FJ: Ammonium assimilation in cyanobacteria. Photosynth Res 2005, 83:135-50.

27. Heinrich A, Maheswaran M, Ruppert U, Forchhammer K: The Synechococcus elongatus $\mathbf{P}_{\mathrm{II}}$ signal transduction protein controls arginine synthesis by complex formation with N-acetyl-L-glutamate kinase. Mol Microbiol 2004, 52:1303-1314. 
28. Burillo S, Luque I, Fuentes I, Contreras A: Interactions between the nitrogen signal transduction protein PII and $\mathrm{N}$-acetyl glutamate kinase in organisms that perform oxygenic photosynthesis. $J$ Bacteriol 2004, 186:3346-3354.

29. Maheswaran M, Urbanke C, Forchhammer K: Complex formation and catalytic activation by the PII signaling protein of $\mathrm{N}$-acetyl-L-glutamate kinase from Synechococcus elongatus strain PCC 7942. J Biol Chem 2004, 279:5520255210.

30. Sugiyama K, Hayakawa $T$, Kudo $T$, Ito $T$, Yamaya $T$ : Interaction of $\mathbf{N}$ acetylglutamate kinase with a $\mathbf{P}_{\mathrm{II}}$-like protein in rice. Plant Cell Physiol 2004, 45:1768-1778.

31. Ferrario-Méry S, Besin E, Pichon O, Meyer C, Hodges M: The regulatory PII protein controls arginine biosynthesis in Arabidopsis. FEBS Lett 2006, 580:2015-2020.

32. Chen YM, Ferrar TS, Lohmeier-Vogel EM, Morrice N, Mizuno Y, Berenger B, Ng KK, Muench DG, Moorhead GB: The PII signal transduction protein of Arabidopsis thaliana forms an arginine-regulated complex with plastid Nacetyl glutamate kinase. J Biol Chem 2006, 281:5726-5733.

33. VanEtten $\mathrm{CH}$, Miller RW, Wolff IA, Jones Q: Amino acid composition of seeds from 200 angiospermous plant species. Agric Food Chem 1963, 5:399-410. 
34. Slocum RD: Genes, enzymes and regulation of arginine biosynthesis in plants. Plant Physiol Biochem 2005, 43:729-745.

35. Maheswaran M, Ziegler K, Lockau W, Hagemann M, Forchhammer K: PIIregulated arginine synthesis controls accumulation of cyanophycin in Synechocystis sp. strain PCC 6803. J Bacteriol 2006, 188:2730-2734.

36. Quintero MJ, Muro-Pastor AM, Herrero A, Flores E: Arginine catabolism in the cyanobacterium Synechocystis sp. Strain PCC 6803 involves the urea cycle and arginase pathway. $J$ Bacteriol 2000, 182:1008-1015.

37. Cunin R, Glansdorff N, Pierard A, Stalon V: Biosynthesis and metabolism of arginine in bacteria. Microbiol Rev 1986, 50:314-352.

38. $\mathrm{Xu}$ Y, Labedan B, Glansdorff $\mathrm{N}$ : Surprising arginine biosynthesis: a reappraisal of the enzymology and evolution of the pathway in microorganisms. Microbiol Mol Biol Rev 2007, 71:36-47.

39. Ramón-Maiques S, Marina A, Gil-Ortiz F, Fita I, Rubio V: Structure of acetylglutamate kinase, a key enzyme for arginine biosynthesis and a prototype for the amino acid kinase enzyme family, during catalysis. Structure 2002, 10:329-342.

40. Gil-Ortiz F, Ramón-Maiques S, Fita I, Rubio V: The course of phosphorus in the reaction of $\mathrm{N}$-acetyl-L-glutamate kinase, determined from the structures of 
crystalline complexes, including a complex with an $\mathrm{AlF}_{4}^{-}$transition state mimic. J Mol Biol 2003, 331:231-244.

41. Fernández-Murga ML, Gil-Ortiz F, Llácer JL, Rubio V: Arginine biosynthesis in Thermotoga maritima. Characterization of the arginine-sensitive $\mathrm{N}$-acetyl-Lglutamate kinase. J Bacteriol 2004, 186:6142-6149.

42. Lohmeier-Vogel EM, Loukanina N, Ferrar TS, Moorhead GB, Thorpe TA: N-acetyl glutamate kinase from Daucus carota suspension cultures: embryogenic expression profile, purification and characterization. Plant Physiol Biochem 2005, 43:854-861.

43. Ramón-Maiques S, Fernández-Murga ML, Gil-Ortiz F, Vagin A, Fita I, Rubio V: Structural bases of feed-back control of arginine biosynthesis, revealed by the structures of two hexameric $\mathrm{N}$-acetylglutamate kinases, from Thermotoga maritima and Pseudomonas aeruginosa. J Mol Biol 2006, 356:695-713.

44. Fernández-Murga ML, Rubio V: Basis of arginine sensitivity of microbial N-

- $\quad$ acetyl-L-glutamate kinases: mutagenesis and protein engineering study with the Pseudomonas aeruginosa and Escherichia coli enzymes. J Bacteriol 2008, 190:3018-3025.

This study of the arginine-sensitive NAGK of Pseudomonas aeruginosa explores the structural determinants of the arginine inhibition of hexameric NAGKs by mutating the arginine site or by mutating residues or deleting parts of the N-helix. The results 
demonstrate the extreme importance of the hexameric organization and of the cross-talk between the arginine sites of different NAGK subunits mediated by the N-helix, for rendering the inhibition sigmoidal and appropriate in terms of sensitivity to the physiological range of arginine concentrations. The results highlight the importance of the stabilization of high or low affinity forms of the arginine site in the determination of the apparent affinity of the enzyme for arginine.

45. Hsieh MH, Lam HM, van de Loo FJ, Coruzzi G: A PII-like protein in Arabidopsis: putative role in nitrogen sensing. Proc Natl Acad Sci U S A 1998, 95:13965-13970.

46. Perutz M: Mechanisms of cooperativity and allosteric regulation in proteins. Cambridge University Press; 1990.

47. Smith CS, Weljie AM, Moorhead GB: Molecular properties of the putative nitrogen sensor PII from Arabidopsis thaliana. Plant J 2003, 33:353-360.

48. Lancien M, Gadal P, Hodges M: Enzyme redundancy and the importance of 2oxoglutarate in higher plant ammonium assimilation. Plant Physiol 2000, 23:817-824.

49. Osanai $\mathrm{T}$, Tanaka K: Keeping in touch with PII: PII-interacting proteins in unicellular cyanobacteria. Plant Cell Physiol 2007, 48:908-914. 


\section{Figure legends}

Figure 1. Roles of PII in ammonia assimilation. (a) Scheme of ammonia assimilation as arginine in oxygenic photosynthetic organisms. Ammonia, the glutamate product of the GS/GOGAT cycle, and the final product arginine are in large type and coloured blue. The red arrow and the red negative sign within a circle symbolize arginine inhibition of NAGK. PII is also shown in large letters and is coloured green. The green arrows and the green positive and negative signs within circles symbolize, respectively, NAGK activation by PII and release of NAGK from feed-back inhibition by arginine. (b) Complex of the Escherichia coli PII protein GlnK with its target AmtB, which is a trimeric ammonia channel (PDB file 2nuu). (c) Zoom of the T-loop interaction with AmtB. (d) Longitudinal section of the ammonia channel of one AmtB subunit, to illustrate the blocking of the channel by the side chain of the T-loop residue Arg47.

Figure 2. NAGK structures and inhibition by arginine. (a) Dimeric, arginine-insensitive E. coli NAGK (PDB file 1gs5) with AMPPNP (an inert ATP analog) and NAG bound, respectively, to the C-domain (in grey) and N-domain (golden) of each subunit. (b) Hexameric, arginine-sensitive NAGK of Thermotoga maritima with bound arginine (PDB file 2bty). Three subunits related by threefold symmetry are shown in grey, whereas the other three subunits are coloured yellow. (c) Scheme illustrating the arginine-triggered movement of the three dimers preserving the molecular NAGK symmetry, and resulting in an increase in the distance between the substrate sites in each subunit. (d) High and (e) low tilt forms of the NAGK dimers in the respective hexameric NAGKs of Pseudomonas aeruginosa (PDB file 2buf) without arginine and in the arginine-containing (arginine molecules are shown in spheres representation) $T$. maritima enzyme. In both cases two of the three dimers forming the ring are shown, in a 
view in which the threefold axis is vertical and in which the molecule is viewed along the twofold molecular axis from the side where two canonical dimers are linked by the N-helices. In each dimer one subunit is grey and the other golden. (f) Sigmoidal arginine inhibition kinetics of Synechococcus elongatus NAGK, illustrating the effects of addition of a saturating amount of PII on enzyme activity and on arginine inhibition. The data correspond to our own unpublished results.

Figure 3. Structure of PII to illustrate various conformations of the T-loop. (a) View of the E. coli PII protein GlnK containing bound ADP, taken from the complex with AmtB (PDB file 2nuu). The threefold axis is vertical and each subunit is in a different colour. A black dot in the T-loop shows the residue that is uridylylated (b) View as in (a) of Arabidopsis thaliana PII bound to MgATP, taken from the complex with NAGK (PDB file 2rd5). A black dot in the T-loop shows the Ser residue corresponding to the Ser that is phosphorylated in cyanobacteria (c) Superimposition of the T-loops of different PII structures. The T-loop in the structures of, (reddish) free PII from S. elongatus (PDB file 1qy7), GlnK from E. coli (violet) in the complex with AmtB (PDB file 2nuu), GlnK of Methanococcus jannaschii with ADP (green, PDB file 2j9d) or with MgATP and 2oxoglutarate (golden, PDB file 2j9e), and PII of $S$. elongatus (blue, PDB file 2v5h) or A. thaliana (cyan, PDB file 2rd5) in their complexes with NAGK. The general orientation, although similar to that in (a), has been modified for optimal viewing of all the superimposed T-loops. (d) The binding of MgATP and 2 oxoglutarate (2OG) in the T-loop of GlnK from M. jannaschii. The orientation is the same as in (b).

Figure 4. The PII-NAGK complex. (a) Structure of the complex of S. elongatus, viewed along a twofold axis. The threefold axis is vertical. Each PII trimer has a different 
shading of yellow, whereas each NAGK dimer is in a different colour. Bound NAG molecules are represented with spheres. Note the high tilt of the dimers, comparable to that in Figure 2e. (b) and (c) Surface representation of the faces that participate in the interaction of, respectively, NAGK and PII, viewed along the threefold axis. Color coding of NAGK is as in (a), and the three subunits of PII are in different shades of yellow. The surfaces for which the accessibility is restricted in the complex are depicted in blue, reddish and deep green, for the T-loop, B-loop and $\beta 1-\alpha 1$ connection of PII and their corresponding complementary surfaces in NAGK. (d) Representation of one NAGK subunit of the A. thaliana complex and of the parts of the PII subunit that participate in the interaction with it, to illustrate the relative positions of the active centre (shown in the more closed conformation, and occupied by NAG and ADP), of the arginine site (with bound arginine), and of the PII elements participating in the interactions between both proteins. (e) Detail of the interactions of the T-loop (backbone in yellow) with the NAGK subunit (in thin trace, with some side-chains in grey) of the PII-NAGK complex of $S$. elongatus. Bound NAG and the $\beta 3-\beta 4$ hairpin that is the mobile lid of the NAG site are shown. $\alpha \mathrm{E}$ and $\alpha \mathrm{G}$ refer to helices of the $\alpha 4$ layer of the NAGK subunit. (f-h) Semitransparent surface representations of the arginine sites in the NAGKs of T. maritima (f) and of the PII-NAGK complexes of $A$. thaliana (g) and S. elongatus (h). Note the change relative to Tyr15, in the N-helix of the high-affinity arginine site of T. maritima NAGK (f), of Phe29 and Tyr23 in (g) and (h), respectively. The change is accompanied by a partial rearrangement of the N-helix. 


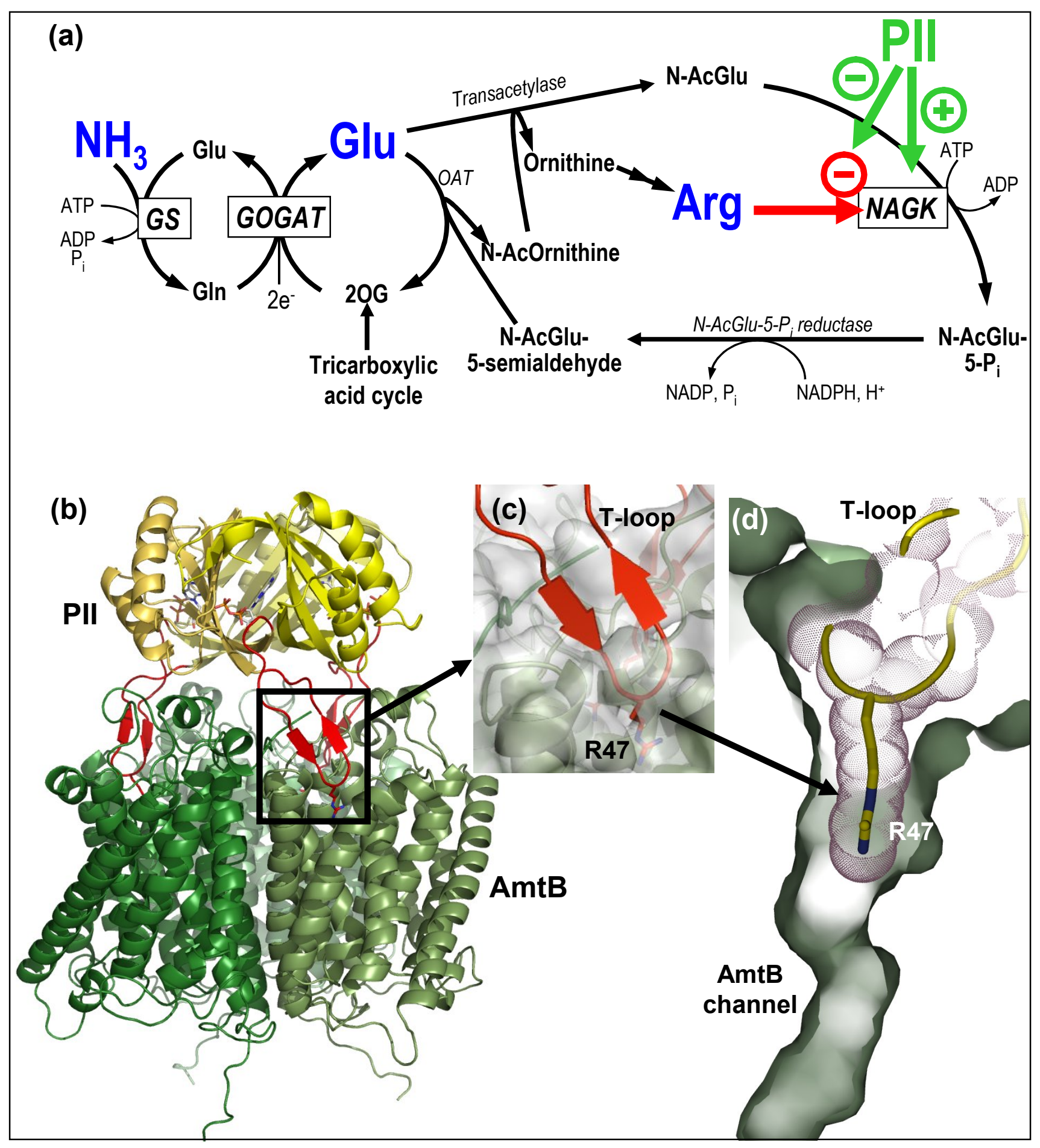

Figure 1 


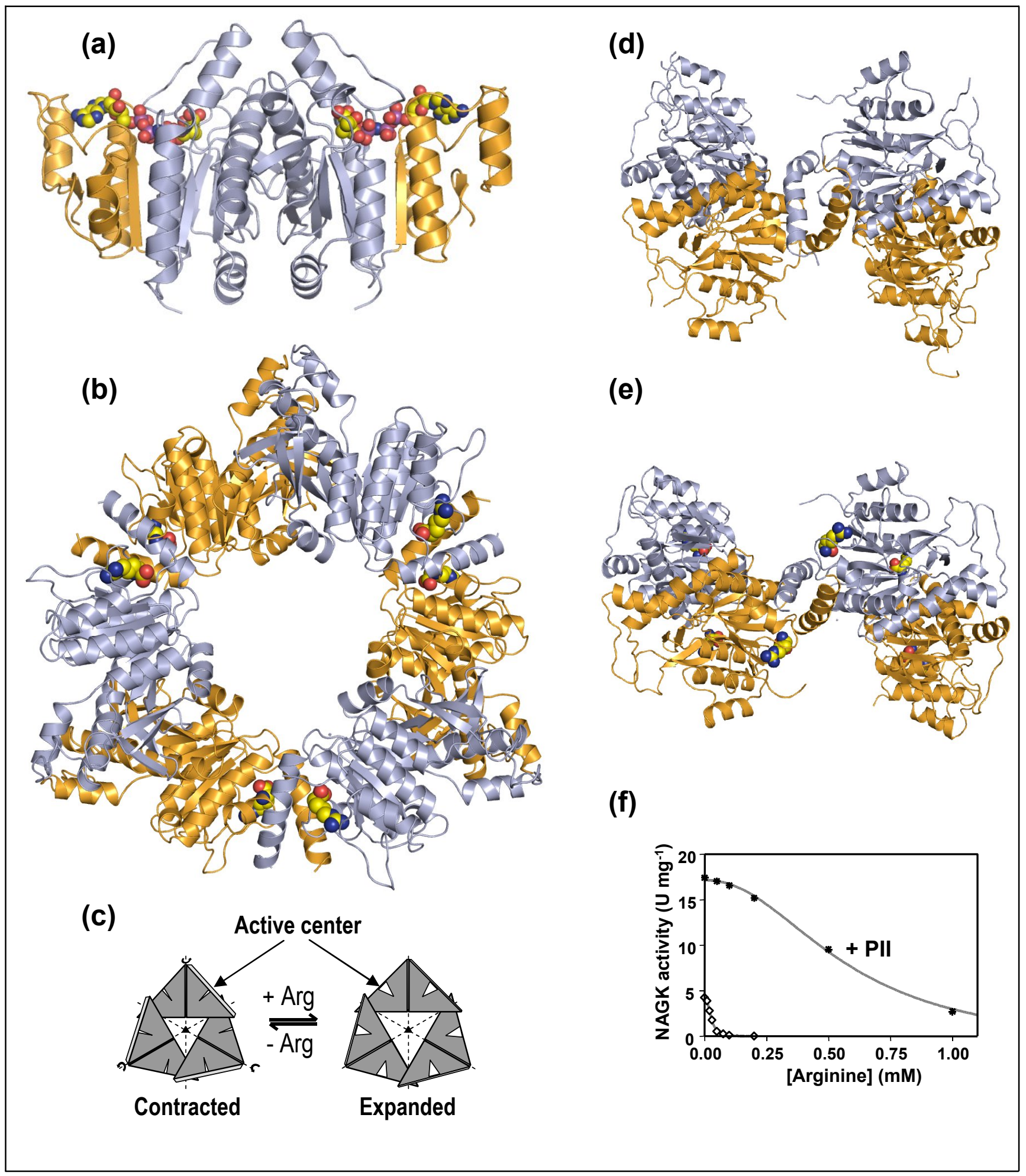

Figure 2 


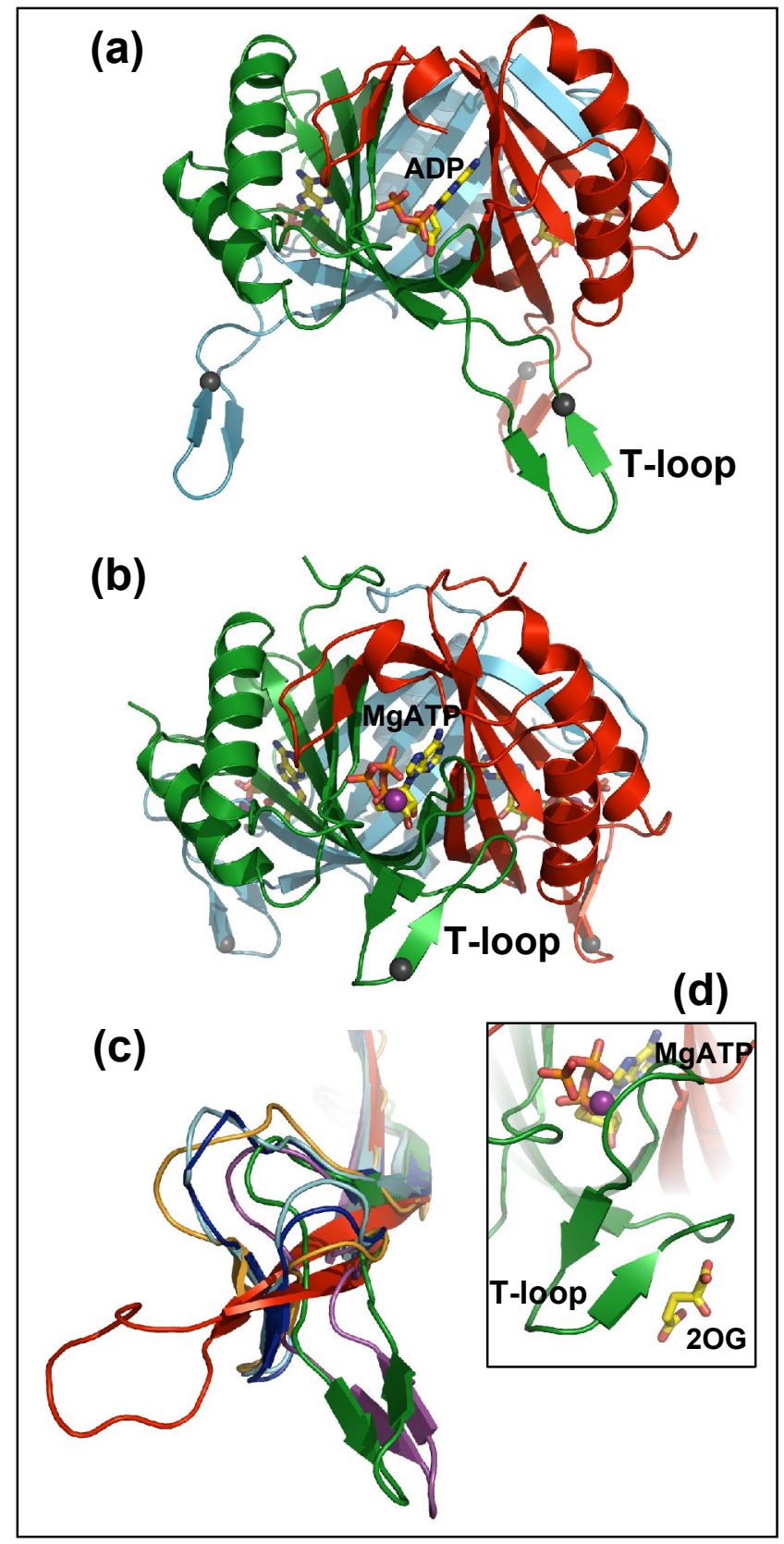

Figure 3 


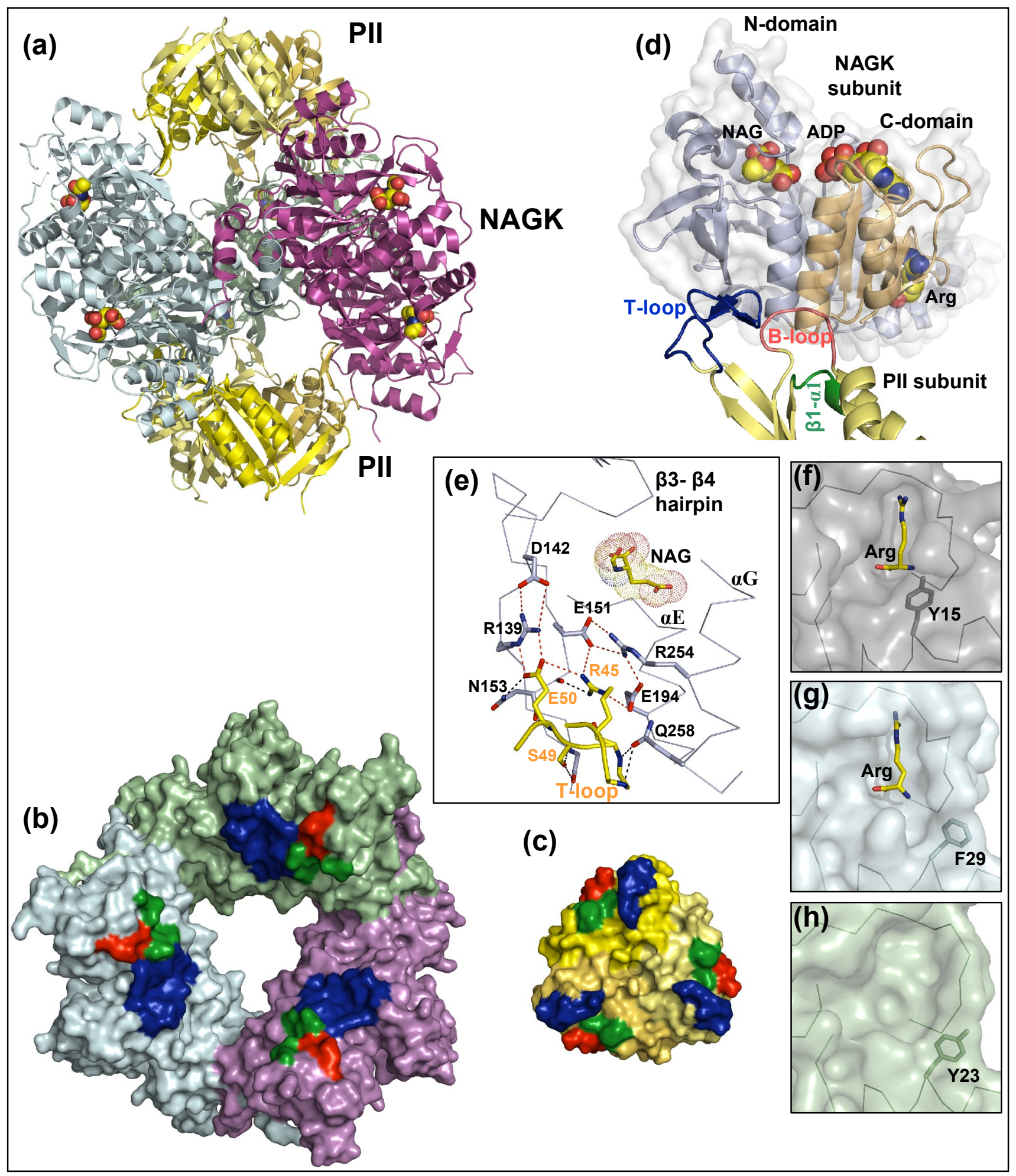

Figure 4 